\title{
The Elusive Search for Interoperability
}

\author{
W. Ed Hammond ${ }^{1}$
}

${ }^{1}$ Duke Center for Health Informatics, Duke University

\begin{abstract}
With the arrival of the information age and transition towards Electronic Health Records (EHR) and Digital Data, the need for aggregating data across multiple sources lead to the concepts of interoperability. Initially, interoperability was defined only from the perspective of technical interoperability and semantic interoperability. Over time, what was required to make things work together expanded the concepts of the scope of this topic. Unfortunately, the momentum of what currently exists, lack of motivation to change, the cost of change, and lack of a clear Return on Investment (ROI), and unclear solutions has made interoperability seemingly an impossible goal. This paper postulates that the definition of interoperability varies based on use case.
\end{abstract}

\section{Correspondence to:}

W. Ed Hammond, Ph.D.

Duke University, Duke Center for Health Informatics

Address: Hock Plaza, 2424 Erwin Road, Suite 907

Durham, North Carolina, 27705 U.S.A.

E-mail: william.hammond@duke.edu
The paper discusses what adjectives the term interoperability might legitimately carry - total, partial, implied,

.. The paper also discusses the problems associated with a focus on the word interoperability and attempting to create standards that enable the concept rather than a focus on what we are really trying to do and then looking at what is required to make that happen. Finally, the paper discusses the recent Request for Information (RFI) from the U.S. Office of the National Coordination (ONC) for Health Information Technology to provide suggestions about how interoperability might be measured.

\section{Keywords}

Interoperability; Health Data Standards; Electronic Health Record

\section{EJBI 2016; 12(1):en15-en18}

received: April 24, 2016

accepted: April 26, 2016

published: May 20, 2016

\section{Introduction}

As computers began to be used in the health care setting, the need to integrate data from multiple sources became desirable. At first, each system created its own interfaces among the sources and at significant cost. Further, with the implementation of new features and new sites, the interfaces had to be maintained and updated at considerable costs. Health Level 7 (HL7) came into being in an effort to create a reusable solution to this problem. HL7's initial focus was to create a standard that would support the development of a Hospital Information System (HIS) from functional components developed by multiple systems. These components were selected from a larger set and represented what different groups identified as "Best of Breed" components. The focus of the standard was to create a message that was the mechanism by which data was transferred from one functional component to another. The functions connected were largely service functions such as clinical laboratory; admission, discharge, transfer; radiology; pharmacy; scheduling; and billing. Next the standards created were designed to support both in-patient and outpatient settings; bed-side instruments; images; and some decision support applica- tions. Even so, these standards were developed largely by technical people and did not involve the clinical community, clinical professional organizations, or government groups. The word interoperability was introduced to define what standards were supposed to do. The Institute of Electrical and Electronic Engineers (IEEE) provided a definition of interoperability that was accepted by the standards community. That definition was "the ability of two or more systems or components to exchange information (identified as functional interoperability) and to use the information that has been exchanged [1 (identified as semantic interoperability)". I would point out that the word information should be replaced by data, since that was what was exchanged. The definition seemed to be adequate as long as the purpose and use of the standard was limited in both scope and stakeholders.

\subsection{Definition of Interoperability}

Over time, as the use of digital data with the health care system expanded to involve more and diverse people and more and diverse purposes, we began to realize that more than just functional and semantic interoperability was required. Additional technical requirements 
appeared in the form of networks, database architecture, structured and non-structured data, privacy and security, legal and propriety issues, patient consent, provenance, triggers, what to exchange, and other drivers. The targets for data exchange continued to expand. The concept of a patient-centric electronic health record in which all data created for and about a patient were aggregated into a single record has evolved. The concept of a Health Information Interchange required standards to support the aggregation of centralized or federated databases for regions of various sizes including states or even countries. The support of sharing data across multiple sites and regional groupings became desirable. The definition of interoperability now became less precise, and what was required for interoperability was less clear.

The current fuzziness of the word interoperability can be realized by simply "Googling" the word. Healthcare Information and Management Systems Society (HIMSS) defines interoperability as "the ability of different information technology systems and software applications to communicate, exchange data, and use the information that has been exchanged." 2] Data exchange schema and standards should permit data to be shared across clinicians, lab, hospital, pharmacy, and patient regardless of the application or application vendor. Interoperability means the ability of health information systems to work together within and across organizational boundaries in order to advance the effective delivery of healthcare for individuals and communities. HIMSS defines three levels of technical interoperability: functional, structural, and semantic. The meaning of functional and semantic is consistent with IEEE. Structural interoperability defines the structure or format of the data exchange. Another, perhaps clearer term is syntactical interoperability.

A comment from the group developing these definitions best expresses the problems: "Interoperability is one of those terms everybody thinks they understand. When you press people for a definition you usually get a shuffling of the feet and a blank look. Well, it's when things talk to each other, right?"

ONC defines interoperability as: "All individuals, their families, and their health care providers have appropriate access to health information that facilitates informed decision-making, supports coordinated health management, allows patients to be active partners in their health and care, and improves the overall health of our population." 3

Wikipedia defines interoperability as "a property of a product or system, whose interfaces are completely understood, to work with other products or systems, present or future, without any restricted access or implementation."

More generally now, interoperability must include social, political, and organizational factors.

\subsection{Personal Experience with Interoperability}

My group at Duke designed our first automated medical record (AMR) (what we called it then) in 1970. Initially, the sites of use were Obstetrics, Primary Care Clinic, and a Renal Dialysis Unit. Each system was independent of the others, but we created a single program, and the differences in the sites were accommodated through a data dictionary. To capture lab data for the AMR a human operator looked at the Lab system terminal and typed the data into the AMR. The error rate was high. There were many different sources of lab data at Duke, so to accommodate an interoperable interface with all, we created our first data interchange standard. The error rate was very high. The standard identified what data items were sent, the format and the syntax of the exchange. Since the terminology used to identify the data items was local, we had to map from the lab terminology to the AMR terminology. The lab names seemed to constantly change, and synchronization of the names - a condition for interoperability - seemed impossible. We participated in the creation of HL7's v2.n standard and early on transitioned from our local standard to the HL7 v2 standard. If we could not process an incoming standard, we moved the message into an error file where a human resolved the problem. In $80 \%$ of the errors we found they were due to a mismatch between what the lab sent and our ability to map it into our AMR terminology. So, even then, interoperability was an elusive goal.

We later evolved into an in-patient setting, and we added bedside monitoring into the functions supported. In this case, we had bit streams from various bedside instruments which we then had to decode, understand, map into our terminology set, and integrate into the AMR. Our first challenge was an instrument data flow that immediately turned off when activated. After lots of frustration, we finally discovered that the first byte transmitted in the data stream was the X-OFF pattern. We then had to recognize that byte and change it to activate the byte-oriented data stream. In this case, interoperability required understanding and accommodating the actual bit-stream coming into the system.

One more example occurred when we implemented the AMR (now identified as The Medical Record (TMR)) in a long term care (LTC) facility. Most patients were admitted into the nursing home from a hospital, and we wanted to transfer data from the hospital into the record of the LTC facility. In this case, we had to first identify what data elements we needed to transfer from the hospital to the LTC as well as map from the hospital local terminology into the LTC local terminology. We then discovered that frequently, patients from the LTC were readmitted back into the hospital, and the process was reversed. But the hospital wanted different data elements from the LTC so a different transfer protocol had to be established.

Two similar use cases were encountered when Family Medicine Department started seeing and taking care 
of women who became pregnant. These women would deliver at Duke OB and may in fact have visits in the OB clinic if issues arose during pregnancy. The family medicine clinic and the obstetrics clinic used different data dictionaries, although the root EHR was the same. In this case, the records for the two systems had to be synchronized, with data flowing bi-directionally between the two systems. The architecture of the two EHR implementations was different, so not only mapping was required between the terminologies, but differences in format and content of the EHR had to be accommodated. Another similar example was creating the pediatric EHR automatically from the mother's OB EHR. Again two different terminologies were mapped and different data architectures were mapped.

More recently, I became involved with a collaborative research project sponsored the Patient Centered Outcomes Research Institute (PCORI) called PCORnet. In this research, queries are made across multiple datamarts aggregated through Clinical Data Research Networks (CDRN). CDRNs are made up of many different institutions and sites. For example, one CDRN, called Greater Plains Collaborative, is composed of 12 leading medical centers across 8 states and extends from Wisconsin to Texas. A Common Data Model was created and is used to define a common set of data elements for the queries. Each participant must map from local representations of data into the common model. The problem is further complicated in that sites include a mixture of claims based data and clinical data. Value sets differ for many of the data elements and what is available or not and how data are represented varies. In this case, establishing interoperability is even more challenging. Different networks, different search engines, different connections and whether CDRNS are centralized or federated may differ across the networks. How will the degree of interoperability be measured under these circumstances? Data characterization is being established by simple queries among the sites (currently 85 datamarts) represented over a million individuals.

Most recently, I have been involved in a pilot study in which data from personal sensors are moved through a series of steps to my personal care provider. Using my Apple Watch and Apple HealthKit, I use watchOS and iOS to move health and fitness data into Epic's MyChart. For my Apple Watch, I automatically capture activity data and heart rate. My scale uses a bluetooth to enter my weight. I enter blood pressure and nutritional data directly into my iPhone. Using SMART on FHIR $\AA$, the data is moved into a flowsheet in MyChart. My PCP then, at times of her choice, looks at the time-oriented data. On two occasions, she has changed my medications as a result of these data. Again, what is required for interoperability has expanded tremendously, and involves multiple stakeholders, many different technologies, networking, people issues, security and privacy, and control.

The purpose of these examples is to show that the meaning of interoperability is a function of the use case.
The meaning includes understanding data representation, solving connectivity issues, mapping terminologies, defining what data is to be exchanged, dealing with an inconsistent mixture of units, dealing with a mixture of data types, and synchronization of data flows among different systems. Issues of privacy, security, unique and essentially error-free patient identification must be resolved. As data interchange broadens, provenance becomes essential. Proprietary vs open systems complicate the exchange of data. Trust and quality become big factors. The mountain of interoperability grows even higher and challenges a solution.

\section{Standards That Support Interoperability}

Where do standards come from? How do standards developing organizations decide what standards they will develop and with what priorities? What is the role of the various stakeholders? What is the role of the government? Do governments mandate, regulate, fund development, encourage, certify, or participate? What is the role of vendors? Do vendors participate and try to influence standards that favorite their products? Does the lack of functional and successful standards make it easier for venders to provide total solutions at a price that once committed, few can afford to change? What role should the user stakeholders play? How do they understand "the art of the possible" to encourage the creation and adoption of standards that add value to the process of healthcare? How do domain experts share their knowledge with the technical developers of standards? I suggest another important question that should be addressed is "Which comes first - standards or requirements?"

In informatics, we frequently define a word, such as interoperability, and fixate on that word rather than on what we are trying to accomplish. We pick our definition and create a standard that addresses that definition. HL7, for example, focuses on designing a standard that provides functional and syntactical interoperability. We overlook semantic interoperability because we are too late to dictate a solution, so we create a standard that accommodates the most widely used terminologies with-in the standard. Have we destroyed any chance to achieve interoperability? Furthermore, there are many other issues that must be addressed that are beyond the scope of HL7.

\section{Discussion}

The U.S. Office of the National Coordinator for Health Information Technology recently issued a Request for Information requesting information about how interoperability might be measured or at least evaluated. The purpose of this paper is to argue that such a task might be impossible, but importantly is really not important. We put interoperability up front and design standards that 
try to accomplish this goal. We hope issues for which we have no solutions, or solutions that we are unable to enforce or get acceptance will somehow magically disappear. We spend time and resources creating work-arounds that continue to cost time and money and the problem never disappears.

Two examples illustrate this point. The first is perhaps doable, if we are willing to make it happen. The issue is unique patient identifiers. At least $80 \%$ of EHRs will contain someone else's data. We have developed a number of algorithms to create a unique identifier from a set of personal identifiers, many of which are chosen from the HIPAA Personal Health Identifiers (PHI) data elements. Validation of this method is based on the presence of the data elements used to create the identifier. The identity error rate, in the absence of a universal personal identifier, is sufficient to limit the ability to create a medication history across multiple settings. Pragmatic clinical trials are likely to be biased by duplicative patient records, some of which may be within the same institution.

The second obvious barrier to interoperability is a single common terminology, used world-wide. Existing terminologies were created for the most part for various purposes, and do not represent with sufficient granularity and precision clinical concepts. The use for financial purposes tends to dominate what is defined. New categories of data, such as biomarkers, genomics, patient-reported data, environmental data, behavioral data, and other data types are not included. Further, the full set of attributes is not specified, and even if they are specified, users ignore them. FHIR has the potential to address some of these problems by starting with fully specified data elements, fully specified resources, and profiles for stated purposes.

\section{Conclusions}

The potential for informatics to make a significant impact on the health of the population of the world could never be greater. Current initiatives in the U.S. include Population Health, Precision Medicine, Learning Health Systems, Big Data, Clinical and Translational Science Awards (CTSA), data sharing, PCORI, ONC, NIH initiatives, and many others. All require the ability to move, share and use data. As evidenced in this paper, the specifics of what is required and the expanse of what is required vary considerably. I suggest that we should first focus on what we want to do - truly visionary - and then create systems, standards, and tools that enable that vision. We also need to bring the community together in creating necessary standards, quick acceptance and implementation, and global use. Any barrier that needs to be overcome should become a primary concern, and energy directed toward removing the barrier - whether it is political, workflow related, people related, or technical. The world is in constant change. We must design accordingly.

\section{References}

[1] IEEE Standard Computer Dictionary: A Compliation of IEEE Standard Computer Glossaries. 1990. New York, NY.

[2] HIMSS Board. April 5, 2013. www.HIMSS.org Accessed April 4, 2016.

[3] www.ONC.org Accessed April 15, 2016. 